\title{
Article \\ Falling from Rest: Particle Creation in a Dropped Cavity
}

\author{
Francesco Sorge (1)
}

I.N.F.N. Sezione di Napoli, Complesso Universitario di Monte S. Angelo, Via Cintia, Edificio 6, I-80126 Napoli, Italy; sorge@na.infn.it

\begin{abstract}
We discuss the process of particle creation in the case of a scalar quantum field confined to a small cavity, initially at rest, which is suddenly dropped in a static gravitational field. We show that, due to the transition from a Schwarzschild to a Minkowski background, as perceived by a comoving observer, field particles are excited out of the quantum vacuum. The density of the created quanta depends on the proper gravitational acceleration as well as on a parameter $\alpha \simeq 1 / \Delta t$, with $\Delta t$ representing the typical time duration of the transition. For the specific acceleration profile considered, the energy spectrum of the created quanta roughly resembles a two-dimensional Planckian distribution, whose equivalent temperature mimics the Hawking-Unruh temperature, with the detector acceleration (or the black hole surface gravity) replaced by the parameter $c \alpha$. We briefly comment on possible issues related to local Lorentz symmetry.
\end{abstract}

Keywords: acceleration; particle creation; quantum fields; general relativity

\section{Introduction}

One of the most striking predictions of quantum theory in curved spacetimes is that

check for updates

Citation: Sorge, F. Falling from Rest: Particle Creation in a Dropped Cavity. Symmetry 2021, 13, 1139. https:// doi.org/10.3390/sym13071139

Academic Editor: Ignatios Antoniadis

Received: 24 May 2021

Accepted: 22 June 2021

Published: 25 June 2021

Publisher's Note: MDPI stays neutral with regard to jurisdictional claims in published maps and institutional affiliations. particles can be created by time-dependent gravitational fields (see, e.g., the pioneering work by Parker [1]) or also in presence of time-independent spacetime backgrounds admitting horizons. On general grounds, particle creation is closely related to the lack of a global timelike Killing vector of the background spacetime. This implies that one cannot define a vacuum state of the theory following a canonical approach, since the vacuum is not invariant under diffeomorfisms. As a consequence, the definition of a vacuum state in a curved spacetime-as well as the very concept of particle-is not unique but observer-dependent.

Hawking radiation [2] and the closely related Unruh [3] radiation are probably the most famous effects concerning the issue of particle creation, although we have so far no direct experimental evidence of either of them [4,5]. On the other hand, it is well-known that the process of particle creation out of a vacuum is not an exclusive feature pertaining to nontrivial background geometries. Particles can be generated due to nonadiabatic changes in the modes of a confined quantum field, resulting either from the motion of the material boundaries constraining the field [6] or as a consequence of a time-dependent properties of the medium in which the field propagates $[7,8]$.

Such processes of particle creation are often classified under the common definition of Dynamical Casimir effect (DCE), although, generally speaking, there is no close relationship with the well-known static Casimir effect, apart from the usual requirement of field confinement (see the excellent review by Dodonov [9] for an almost complete overview of the theoretical as well as the experimental state of the art about the DCE).

Concerning the DCE, DeWitt [10] and Davies and Fulling [11,12] have described particle production due to a moving mirror, acting as an accelerated boundary. Recently, Good et al. [13] have investigated the effect of particle creation, taking into account the rather unexplored aspect related to the time dependence of the particle production process.

In some cases, the time-dependent properties of the medium in which the quantum field propagates can be encoded in the background geometry of an otherwise flat spacetime, 
following an approach based on the so-called Gordon metric. In that respect, particle creation induced by time-dependent boundaries or media can be described in a rather unified way, also in the presence of a curved background (see, e.g., [14]).

When discussing particle creation, a common approach is to define, where possible, suitable in- and out- vacuum states, subsequently finding the adequate Bogolubov transformation relating the field operators acting on the corresponding in- and out- Fock spaces [15]. The procedure is almost straightforward in the case the spacetime admits asymptotically flat, minkowskian regions in the far past and future, or-at least-when the in- and out- regions allow a suitable timelike Killing vector.

Particle creation inside a Casimir cavity falling in a Schwarzchild black hole has been recently discussed in [16,17], assuming that the cavity started its fall from the spatial infinity, where the gravitational field is vanishing.

In the present paper we aim to investigate a similar process, involving particle creation of a massless scalar field, confined to a small cavity, when the latter-previously at rest in the field of a nearby static gravitational source-is suddenly left in free fall.

According to an observer comoving with the cavity, the background spacetime suffers a rapid change in time, from Schwarzschild to Minkowski geometry (we neglect-under reasonable assumptions - tidal effects inside the falling cavity). Instead of describing a change of the cavity worldline in a fixed Schwarzschild geometry, we use the Einstein Equivalence Principle (EEP), considering a static cavity in a time-varying background geometry which mimics and encodes the transition from a noninertial frame to an inertial, freely-falling one.

We show that, as a consequence of the time-changing geometry, the vacuum of a massless scalar field confined to the cavity undergoes to the process of particle creation. Finally, we give an estimate of the number density of the created quanta as well as of the energy spectrum. Here are the main findings:

- For the specific acceleration profile considered, the density energy spectrum of the field quanta created inside the cavity recalls a two-dimensional Planckian distribution.

- The equivalent temperature of such a distribution is not controlled by the gravitational acceleration parameter $\gamma_{0}$ (as in the Hawking-Unruh temperature) but by the jerk parameter $c \alpha$ (namely, the second time-derivative of the velocity), related to the rapidity of the cavity transition from its rest state to the free fall.

- The shape of the energy spectrum does depend on the choice of the acceleration profile, $\gamma(t)$. However, it is expected to become less and less sensitive to the details of the function $\gamma(t)$, as the transition time $\Delta t=1 / \alpha \rightarrow 0$.

- In the weak field limit, the energy density of the excited field quanta is proportional to the gravitational field energy density (encoded in the $t t$-component of the stressenergy pseudotensor $\left.t_{\mu v}\right)$ through the quantity $\left(t_{P} / \Delta t\right)^{2}$, with $t_{P}$ being the Planck time. Thus, the ratio $\left(t_{P} / \Delta t\right)$ measures - so to say-the degree of efficiency in converting the gravitational field energy into the energy of the quanta excited inside the cavity. This latter result deserves further investigation.

The paper is organized as follows.

In Section 2 we briefly review the Klein-Gordon equation in Fermi coordinates for a massless scalar field, confined to a small cavity. In Section 3 we introduce a suitable model for a dropped cavity. In Section 4 we perturbatively solve the Klein-Gordon equation for the scalar field. In Section 5 we compute the $\beta$-Bogolubov coefficients related to the process of particle creation, hence evaluating the number density and the energy density of the created field quanta. Finally, in Section 6 we summarize and discuss the main results of the present analysis, also briefly commenting on possible issues related to local Lorentz symmetry.

Throughout the paper, unless otherwise specified, use has been made of natural units: $c=1, \hbar=1, G=1$. Greek indices take values from 0 to 3; Latin ones take values from 1 to 3 . The metric signature is -2 , with determinant $g$. 


\section{Scalar Field in a Cavity}

Let $\phi(x)$ be a massless scalar field confined to a small cavity. We will model the cavity as in the Casimir effect, considering two parallel plates of proper area $A_{\perp}$, whose constant proper separation is $L$ and assuming that $\sqrt{A_{\perp}} \ll L$. A constant separation means that the cavity is rigid. Suppose that the cavity is placed at rest in a static gravitational field. Although for our purposes it is not essential to know the explicit form of the background metric, for sake of clarity we will take the latter to be the Schwarzschild one, due to a gravitational source of mass $M$

$$
d s^{2}=\left(1-\frac{2 M}{r}\right) d t^{2}-\left(1-\frac{2 M}{r}\right)^{-1} d r^{2}-r^{2}\left(d \theta^{2}+\sin ^{2} \theta d \phi^{2}\right) .
$$

We will suppose that the cavity plates are orthogonal to the gravitational acceleration. Let us introduce a Fermi reference frame $\{t, x, y, z\}$ [18-20], having the origin $\mathrm{O}$ on the lower cavity plate. In the neighborhood of $\mathrm{O}$, the spatial coordinates $x, y, z$ span the axes of a Fermi-Walker triad, $t$ being the proper time. Up to terms linear in the spatial coordinates we have, at a distance $z$ above the lower plate

$$
d s^{2}=\left(1+2 \gamma_{0} z\right) d t^{2}-d x^{2}-d y^{2}-d z^{2}+O\left(|x|^{2}\right),
$$

where $\gamma_{0}=M / R^{2}$ is the gravitational acceleration of the cavity with respect to a locally freely falling observer. Notice that (2) holds true within a world-tube of radius $1 / \gamma_{0}$, so that $\left|\gamma_{0} z\right| \ll 1$. Such condition is safely satisfied for a small cavity, provided $\gamma_{0} L \ll 1$ (see, e.g., Buchholz et al. [21] for a thorough discussion about the rigidity issue).We point out that the present analysis does not strictly require a weak gravitational field. Actually, the condition $\gamma_{0} L \ll 1$ can be satisfied also in presence of a strong acceleration $\gamma_{0}$, provided that the cavity size $L$ is small enough.

The confined field obeys the Klein-Gordon equation with the usual Dirichlet boundary conditions at the cavity plates. For a massless, minimally coupled field, we have

$$
\frac{1}{\sqrt{-g}} \partial_{\mu}\left[\sqrt{-g} g^{\mu v} \partial_{\nu} \phi(x)\right]=0,
$$

along with

$$
\left.\phi(x)\right|_{z=0}=\left.\phi(x)\right|_{z=L}=0 .
$$

In the coordinates (2), (3) reads, up to the first order in $\gamma_{0} z$

$$
\partial_{t}\left[\left(1-\gamma_{0} z\right) \partial_{t} \phi\right]-\left(1+\gamma_{0} z\right) \nabla^{2} \phi-\gamma_{0} \partial_{z} \phi=0 .
$$

\section{A Model for a Dropped Cavity}

Our analysis focuses on the cavity transition from rest to a free fall. From the point of view of an observer comoving with the cavity, this transition appears as a sudden change in the background metric from the form (2) to a flat Minkowski form. In other words, instead of describing a change of the cavity worldline in a fixed Schwarzschild geometry, we use the EEP to discuss a static cavity in a time-varying background, whose geometry mimicks and encodes the transition from a noninertial frame to an inertial, freely-falling one. Such a transition can be described introducing a suitable time-dependent acceleration $\gamma(t)$, whose value changes from $\gamma=\gamma_{0}$ to $\gamma=0$ in a short lapse of time $\Delta t$, required to take the cavity from being at rest to a free fall.

We will assume that the function $\gamma(t)$ behaves smoothly, changing from an initial value $\gamma_{0}$ to a vanishing value of the acceleration (representing the final free fall stage) in a very small lapse of time. The explicit form of $\gamma(t)$ will clearly depend on the physical details of the process leading to the cavity release. However, in order to carry on the calculations in a closed form, let us model the function $\gamma(t)$ as follows 


$$
\gamma(t)=\frac{1}{2} \gamma_{0}(1-\tanh \alpha t) .
$$

In (6) $\alpha \sim \frac{1}{\Delta t}$. If $t \ll-1 / \alpha$, then $\gamma(t) \rightarrow \gamma_{0}$, thus reproducing the initial stage, in which the cavity is at rest in the gravitational field. On the other hand, when $t \gg+1 / \alpha$, $\gamma(t) \rightarrow 0$, hence describing the final stage of the cavity free fall. Notice that in the limit case $\alpha \rightarrow \infty$, representing an instantaneous cavity release, $\gamma(t)$ becomes proportional to the Heaviside step function, $\gamma(t)=\gamma_{0} \Theta(-t)$.

Assuming a time-dependent value of the gravitational acceleration, hence making the replacement $\gamma_{0} \rightarrow \gamma(t)$ in the Klein-Gordon Equation (5), the latter becomes

$$
\square \phi-2 z \gamma(t) \partial_{t}^{2} \phi-\gamma(t) \partial_{z} \phi-z \partial_{t} \gamma(t) \partial_{t} \phi=0,
$$

where $\phi(x)$ obeys the boundary conditions (4).

Notice that we are still using the same coordinates as in (2), but now the "perceived" background metric is time-dependent. The comoving observer experiences a rapid change from an almost uniform gravitational field to a free-fall state, in which gravitational effects do disappear. In the next section we will perturbatively solve the above equation.

\section{Solving the Scalar Field Equation}

Let us start considering the unperturbed $\left(\gamma_{0}=0\right)$ case. The solution of the KleinGordon equation $\square \phi^{(0)}(x)=0$ with the appropriate boundary conditions (4) reads

$$
\phi^{(0)}(x)=N e^{-i \omega\left(k_{\perp}, n\right) t} e^{i \vec{k}_{\perp} \cdot \vec{x}_{\perp}} \sin \left(\frac{n \pi z}{L}\right),
$$

where $N$ is a normalization constant, $\vec{k}_{\perp}=\left(k_{x}, k_{y}\right)$ refers to the transverse mode contribution, $\vec{x}_{\perp}=(x, y)$ and

$$
\omega\left(k_{\perp}, n\right)=\left[k_{\perp}^{2}+\left(\frac{n \pi}{L}\right)^{2}\right]^{\frac{1}{2}}
$$

Up to the first order in the small quantity $\gamma z$, we can recast (7) as

$$
\square \phi(x)=J(x),
$$

where the perturbation in the RHS acts as a source term

$$
\begin{array}{r}
J(x)=\left[\gamma(t)\left(\frac{n \pi}{L}\right) \cos \left(\frac{n \pi z}{L}\right)-i z \omega\left(k_{\perp}, n\right) \partial_{t} \gamma(t) \sin \left(\frac{n \pi z}{L}\right)\right. \\
\left.-2 z \gamma(t) \omega^{2}\left(k_{\perp}, n\right) \sin \left(\frac{n \pi z}{L}\right)\right] N e^{-i \omega\left(k_{\perp}, n\right) t} e^{i \vec{k}_{\perp} \cdot \vec{x}_{\perp}} .
\end{array}
$$

We can write the solution of (10) as

$$
\phi(x)=\phi^{(0)}(x)+\delta \phi(x),
$$

where

$$
\delta \phi(x)=\int_{\Omega} d^{4} x^{\prime} \mathcal{G}\left(x, x^{\prime}\right) J\left(x^{\prime}\right) .
$$

The integration is all over the world tube $\Omega$, whose spatial sections describe the cavity volume. In addition,

$$
\begin{aligned}
\mathcal{G}\left(x, x^{\prime}\right)= & \frac{1}{2 \pi^{2} L} \Theta\left(t-t^{\prime}\right) \sum_{m} \int d^{4} p_{\perp} e^{i \vec{p}_{\perp}\left(\vec{x}_{\perp}-\vec{x}_{\perp}^{\prime}\right)} \frac{\sin \left[\omega\left(p_{\perp}, m\right)\left(t-t^{\prime}\right)\right]}{\omega\left(p_{\perp}, m\right)} \\
& \times \sin \left(\frac{m \pi z}{L}\right) \sin \left(\frac{m \pi z^{\prime}}{L}\right)
\end{aligned}
$$


is the retarded Green function appropriate to the boundary conditions (4). Substituting (11) and (14) in (13) we find, after some straightforward algebra

$$
\begin{array}{r}
\quad \delta \phi(x)=\frac{2 N}{L} \int d t^{\prime} \Theta\left(t-t^{\prime}\right) \sum_{m} e^{\vec{k}_{\perp} \cdot \vec{x}_{\perp}} \frac{\sin \left[\omega\left(k_{\perp}, m\right)\left(t-t^{\prime}\right)\right]}{\omega\left(k_{\perp}, m\right)} I_{m n} \\
\times \sin \left(\frac{m \pi z}{L}\right)\left[-i \omega\left(k_{\perp}, n\right) \partial_{t^{\prime}} \gamma\left(t^{\prime}\right)-2 \omega^{2}\left(k_{\perp}, n\right) \gamma\left(t^{\prime}\right)\right] e^{-i \omega\left(k_{\perp}, n\right) t^{\prime}},
\end{array}
$$

having defined

$$
\begin{aligned}
I_{m n} & =\int_{0}^{L} d z^{\prime} z^{\prime} \sin \left(\frac{m \pi z^{\prime}}{L}\right) \sin \left(\frac{n \pi z^{\prime}}{L}\right) \\
& =\frac{2 L^{2}}{\pi^{2}} \frac{m n}{\left(m^{2}-n^{2}\right)^{2}}[\cos (m \pi) \cos (n \pi)-1] .
\end{aligned}
$$

Notice that the first term in square brackets appearing in (11) yields no contribution upon $z^{\prime}$ - integration in (13). Hereafter we will use a shortened notation in (15), defining $\omega_{m}=\omega\left(k_{\perp}, m\right)$ and $\omega_{n}=\omega\left(k_{\perp}, n\right)$.

We are interested in the final state $t \gg 1 / \alpha$, in which the cavity is in free fall. Taking the $t \rightarrow+\infty$ limit of (15), we obtain

$$
\begin{aligned}
\delta \phi(x)= & \frac{2 N}{L} \int d t^{\prime} \sum_{m} \frac{e^{i \vec{k}_{\perp} \cdot \vec{x}_{\perp}}}{2 i \omega_{m}}\left[e^{i \omega_{m} t} e^{-i \omega_{m} t^{\prime}}-e^{-i \omega_{m} t} e^{i \omega_{m} t^{\prime}}\right] \\
& \times I_{m n} \sin \left(\frac{m \pi z}{L}\right)\left[-i \omega_{n} \partial_{t^{\prime}} \gamma\left(t^{\prime}\right)-2 \omega_{n}^{2} \gamma\left(t^{\prime}\right)\right] e^{-i \omega_{n} t^{\prime}} .
\end{aligned}
$$

Before we proceed, let us discuss the conditions which have to be met in order the present perturbative approach does hold true. Looking at (11) (and recalling that the first term in square brackets does not contribute) we find that $J(x)$ can be considered as a small perturbation provided that

$$
z \gamma_{0} \ll 1, \quad z \partial_{t} \gamma(t) \ll \omega .
$$

Since $\partial_{t} \gamma(t) \simeq \alpha \gamma_{0}$ and $0 \leq z \leq L,(18)$ hold true if

$$
L \gamma_{0} \ll 1, \quad \frac{L \alpha \gamma_{0}}{\omega} \ll 1 .
$$

As previously discussed in the introduction, the first constraint in (19) is already fulfilled in the framework of the Fermi approximation. This is also referred to as the Born rigidity condition [22,23]. Such condition has to be obviously required, in order the cavity can be considered rigid. Furthermore, from $\omega \geq \omega_{\min } \sim \frac{1}{L}$ we have

$$
\frac{L}{\omega}<L^{2} \Rightarrow \frac{L \alpha \gamma_{0}}{\omega}<\alpha \gamma_{0} L^{2}
$$

So, the second constraint in (19) is fulfilled provided that $\alpha \ll \frac{1}{\gamma_{0} L^{2}}$ (or $\alpha \ll \frac{c^{3}}{\gamma_{0} L^{2}}$, in SI units). As a numerical example, for a small cavity whose size is $L=10^{-6} \mathrm{~m}$, we have the following bounds

$$
\gamma_{0} \ll 10^{23} \mathrm{~m} / \mathrm{s}^{2}, \quad \alpha \ll \frac{10^{37}}{\gamma_{0}} \mathrm{~s}^{-1} .
$$

\section{Particle Creation}

The process of particle creation inside the cavity as a consequence of the release of the cavity in the gravitational field is controlled by the $\beta$-Bogolubov coefficient. Equations (12) and (17) together describe the final out- state of the quantum field, when the cavity is in 
free fall. On general grounds (see, e.g., [15], p. 146), the out- modes can be expanded in terms of the in- modes (8) as

$$
\phi_{k}^{\text {out }}(x)=\alpha_{k} \phi_{k}^{\text {in }}(x)+\beta_{k} \phi_{k}^{\text {in } *}(x) .
$$

Strictly speaking, the in- modes $\phi_{k}^{\text {in }}(x)$ are not Minkowski plane waves. Rather, they are distorted, due to the initial background Schwarzschild geometry. However, such a distortion yields an $O(\gamma)$ contribution (cfr. [24]), hence-at the present first-order approximationthe in- modes can be safely replaced by their Minkowski plane-wave counterparts [see Equation (8)]. Comparing (22) with Equations (12)-(17), we see that the $\beta$-Bogolubov coefficient is

$$
\beta_{m n}\left(k_{\perp}\right)=\frac{I_{m n}}{i \omega_{m} L} \int d t^{\prime} e^{-i\left(\omega_{m}+\omega_{n}\right) t^{\prime}}\left[-i \omega_{n} \partial_{t^{\prime}} \gamma\left(t^{\prime}\right)-2 \omega_{n}^{2} \gamma\left(t^{\prime}\right)\right] .
$$

Recalling the explicit form of $\gamma(t)$, [see (6)], we have

$$
\beta_{m n}\left(k_{\perp}\right)=\frac{I_{m n}}{i \omega_{m} L} \int d t^{\prime} e^{-i \omega_{+} t^{\prime}}\left[\frac{i \omega_{n} \gamma_{0} \alpha}{2} \operatorname{sech}^{2} \alpha t^{\prime}+\omega_{n}^{2} \gamma_{0} \tanh \alpha t^{\prime}-\omega_{n}^{2} \gamma_{0}\right]
$$

where $\omega_{+}=\omega_{m}+\omega_{n}$. The last term gives no contribution, being proportional to $\delta\left(\omega_{+}\right)=0$. Performing the remaining integrations, we obtain

$$
\beta_{m n}\left(k_{\perp}\right)=\frac{\pi \gamma_{0} I_{m n}}{2 \alpha L} \frac{\omega_{n}}{\omega_{m}}\left(\omega_{n}-\omega_{m}\right) \frac{1}{\sinh \left(\frac{\pi\left(\omega_{m}+\omega_{n}\right)}{2 \alpha}\right)} .
$$

In (25) the $\beta$-Bogolubov coefficient depends on the continuous value $\vec{k}_{\perp}$ of the transverse momentum as well as on the discrete value of the longitudinal momentum, $k_{\|}=\frac{n \pi}{L}$, via the quantum number $n$. Let us rewrite $\omega_{n}$ and $\omega_{m}$ as

$$
\omega_{n}=\frac{\pi}{L} \sqrt{\xi^{2}+n^{2}}, \quad \omega_{m}=\frac{\pi}{L} \sqrt{\xi^{2}+m^{2}}, \quad \xi=\frac{k_{\perp} L}{\pi} .
$$

Define

$$
\chi=\sqrt{\xi^{2}+n^{2}}+\sqrt{\xi^{2}+m^{2}}
$$

so that

$$
\omega_{+}=\omega_{n}+\omega_{m}=\frac{\pi}{L} \chi
$$

Squaring (28)

$$
\omega_{n}^{2}+\omega_{m}^{2}+2 \omega_{n} \omega_{m}=\frac{\pi^{2}}{L^{2}} \chi^{2}
$$

Furthermore, from (26)

$$
\omega_{n}^{2}-\omega_{m}^{2}=\frac{\pi^{2}}{L^{2}}\left(n^{2}-m^{2}\right) .
$$

Combining (29) and (30) we obtain

$$
\begin{aligned}
& \omega_{n}=\frac{\pi}{2 L} \frac{\chi^{2}+n^{2}-m^{2}}{\chi}, \\
& \omega_{m}=\frac{\pi}{2 L} \frac{\chi^{2}-n^{2}+m^{2}}{\chi} .
\end{aligned}
$$

Substituting in (25) and using also (16) we get

$$
\beta_{m n}(\chi)=\frac{\gamma_{0}}{\alpha} \frac{m n[\cos (m \pi) \cos (n \pi)-1]}{m^{2}-n^{2}} \frac{\chi^{2}+n^{2}-m^{2}}{\chi\left(\chi^{2}-n^{2}+m^{2}\right)} \sinh ^{-1}\left(\frac{\pi^{2}}{2 \alpha L} \chi\right) .
$$


Equation (33) explicitly shows the dependence of the $\beta$-Bogolubov coefficient on on the continuum $(\chi)$ as well as on the discrete $(n, m)$ variables, describing the degrees of freedom of the field modes inside the cavity.

\subsection{Density $\varrho_{n}$ of Created Quanta}

The density of field particles created in the state $n$ is [15]

$$
\varrho_{n}=\frac{\pi}{L} \sum_{m} \int d^{2} k_{\perp}\left|\beta_{m n}\left(k_{\perp}\right)\right|^{2} .
$$

Inspection of (25) shows that $\beta_{n n}\left(k_{\perp}\right)=0$. Actually, $\lim _{m \rightarrow n} I_{m n}=L^{2} / 4$. Furthermore, for any fixed value of $n, I_{m n}$ is strongly peaked around $m=n$, while $I_{m n}=0$ if $m=n \pm 2 p, p \in N$. Hence, the dominant terms contributing to field quanta excitation in the state $n$ are those with $m=n \pm 1$. Thus, within a good approximation

$$
\varrho_{n} \simeq \frac{\pi}{L} \sum_{m=n \pm 1} \int d^{2} k_{\perp}\left|\beta_{m n}\left(k_{\perp}\right)\right|^{2}
$$

Differentiating (27)

$$
d \chi=\frac{L}{\pi} \frac{\omega_{n}+\omega_{m}}{\omega_{n} \omega_{m}} k_{\perp} d k_{\perp}
$$

and using (31) we have

$$
k_{\perp} d k_{\perp}=\frac{\pi^{2}}{4 L^{2}} \frac{\left(\chi^{2}+n^{2}-m^{2}\right)\left(\chi^{2}-n^{2}+m^{2}\right)}{\chi^{3}} d \chi .
$$

Placing (33) and (37) in (35) we finally find the result

$$
\varrho_{n}=\frac{2 \pi^{4} \gamma_{0}^{2}}{L^{3} \alpha^{2}} \sum_{m=n \pm 1} \int_{m+n}^{+\infty} d \chi \frac{m^{2} n^{2}}{\left(m^{2}-n^{2}\right)^{2}} \frac{\left(\chi^{2}+n^{2}-m^{2}\right)^{3}}{\chi^{5}\left(\chi^{2}-n^{2}+m^{2}\right)} \sinh ^{-2}\left(\frac{\pi^{2}}{2 \alpha L} \chi\right),
$$

where an overall factor 4 , due to the quantity $[\cos (m \pi) \cos (n \pi)-1]$ in (33), has been taken into account.

\subsection{Energy Density of Created Quanta}

We now move to evaluate the energy density of the created particles. The energy density for the n-th excited state is [15]

$$
u_{n} \simeq \frac{\pi}{L} \sum_{m=n \pm 1} \int d^{2} k_{\perp}\left|\beta_{m n}\left(k_{\perp}\right)\right|^{2} \omega_{m}\left(k_{\perp}\right) .
$$

where use has been made of (35). Following the same steps as above, we obtain

$$
u_{n}=\frac{\pi^{5} \gamma_{0}^{2}}{L^{4} \alpha^{2}} \sum_{m=n \pm 1} \int_{m+n}^{+\infty} d \chi \frac{m^{2} n^{2}}{\left(m^{2}-n^{2}\right)^{2}}\left(1+\frac{n^{2}-m^{2}}{\chi^{2}}\right)^{3} \sinh ^{-2}\left(\frac{\pi^{2}}{2 \alpha L} \chi\right) .
$$

For large values of the quantum number $n(n \gg 1)$ the above cumbersome formula (40) can be approximated as follows

$$
u_{n} \simeq \frac{\pi^{5} \gamma_{0}^{2}}{L^{4} \alpha^{2}} n^{2} \int_{2 n}^{+\infty} d \chi \sinh ^{-2}\left(\frac{\pi^{2}}{2 \alpha L} \chi\right)
$$

Upon integration we have

$$
u_{n} \simeq \frac{2 \pi^{3} \gamma_{0}^{2}}{L^{3} \alpha} n^{2}\left[\operatorname{coth}\left(\frac{\pi^{2} n}{\alpha L}\right)-1\right] .
$$


Notice, however, that the above result roughly holds also for fairly low values of the quantum number $n$, thanks to the rapid convergence of the integrand in (40). We may also recast (42) in the more suggestive form

$$
u_{n} \simeq \frac{4 \pi^{3} \gamma_{0}^{2}}{L^{3} \alpha} \frac{n^{2}}{\exp \left(\frac{2 \pi^{2} n}{\alpha L}\right)-1} .
$$

The total energy density $u$ is recovered summing over the discrete longitudinal modes

$$
u \simeq \frac{4 \pi^{3} \gamma_{0}^{2}}{L^{3} \alpha} \sum_{n} \frac{n^{2}}{\exp \left(\frac{2 \pi^{2} n}{\alpha L}\right)-1} .
$$
we have

Replacing the sum with a continuous integration, i.e., $\sum_{n} \rightarrow \frac{L}{\pi} \int d \epsilon$, where $\epsilon=\frac{n \pi}{L}$,

$$
u=\frac{4 \gamma_{0}^{2}}{\alpha} \int_{0}^{+\infty} d \epsilon \frac{\epsilon^{2}}{\exp \left(\frac{2 \pi \epsilon}{\alpha}\right)-1} .
$$

We thus see that, for the specific acceleration profile (6) we adopted, the energy density of the excited quanta recalls the energy density of a two-dimensional black-body (see, e.g., [25])

$$
u_{b b}(T)=\frac{1}{2 \pi} \int d \epsilon \frac{\epsilon^{2}}{\exp \left(\frac{\epsilon}{k_{B} T}\right)-1},
$$

characterized by an equilibrium temperature

$$
T=\frac{\alpha}{2 \pi k_{B}},
$$

or, in SI units

$$
T=\frac{\hbar \alpha}{2 \pi k_{B}} .
$$

The latter resembles the Hawking-Unruh temperature $T_{\mathrm{HU}}=\frac{\hbar a}{2 \pi c k_{B}}[2,3,26]$. The main difference is that, in the present case, the acceleration $a$ of the Unruh detector (or the black hole gravitational acceleration in the Hawking effect) is replaced by the quantity $c \alpha$.

The apparently odd relationship with a two-dimensional system can be understood noticing that, for each value of $n$, labeling the corresponding state, the transverse vector $\vec{k}_{\perp}$ spans a two-dimensional reciprocal space, $\left(k_{x}, k_{y}\right)$.

Notice also that, for any given $n$, the quantity $u_{n}$ in (42) represents the energy density of a collective excitation mode, involving all the field modes having different $\vec{k}_{\perp}$ values but sharing the same longitudinal component $k_{\|}$. In other words, we are faced with a confined quantum system which can be thought of as made of quasiparticles, whose discrete energy levels are labeled by a single quantum number $n$.

The integration in (45) can be performed in terms of a Riemann Zeta function [27]. The result is

$$
u=\frac{\gamma_{0}^{2} \alpha^{2}}{\pi^{3}} \zeta(3)
$$

which, in SI units, reads

$$
u=\frac{\hbar \gamma_{0}^{2} \alpha^{2}}{\pi^{3} c^{5}} \zeta(3) .
$$

We point out that $u$ depends both on the gravitational acceleration experienced by the cavity and the parameter $\alpha$, related to the transition time $\Delta t$ required to take the cavity from being at rest to the free fall.

There is also-at least in the limit of the weak field approximation, i.e., in the case of a "not so huge" $\gamma_{0}$-an interesting relationship with the gravitational stress-energy 
pseudo-tensor $t_{\mu \nu}$ [28], whose $t t$ component (at the lowest approximation order and in SI units) reads $t_{t t}=\frac{1}{8 \pi G} \gamma_{0}^{2}$. The latter can be considered as the energy density $w_{g} \equiv t_{t t}$ of the gravitational field in which the cavity is immersed. If we rewrite (50) in terms of $w_{g}$, we find (in SI units)

$$
u=\frac{8}{\pi^{2}} \zeta(3)\left(\frac{t_{P}}{\Delta t}\right)^{2} w_{g}
$$

where $t_{P}=\left(\frac{\hbar G}{c^{5}}\right)^{1 / 2}$ is the Planck time. It is well known that the gravitational energy cannot be localized. Actually, it is always possible to choose a reference frame in which the gravitational pseudo-tensor $t_{\mu \nu}$ is locally vanishing. This is exactly what we do when we let the cavity fall: we suddenly move from a reference frame in which $t_{\mu \nu} \neq 0$ to a freely falling (nearly inertial) frame in which $t_{\mu \nu}$ is almost zero. We say "almost", since we are discarding tidal effects [17], which could indeed reveal the presence of a true gravitational field, experienced by the falling cavity.

On the other hand, an observer comoving with the cavity could ask herself where the gravitational energy has gone, after the sudden release of the cavity. Actually, while in the reference frame of an observer at rest with respect to the gravitational source the process of particle creation inside the cavity can be ascribed to the nonadiabatic change in the boundary conditions governing the quantum field modes, according to the comoving observer the creation effect is related to the sudden change in the (local) background geometry, with the subsequent disappearance of the (local) gravitational field. In that respect, (51) seems to suggest that part of the gravitational energy has been used to convert virtual quanta of the scalar field, inhabiting the vacuum inside the cavity, into real field particles whose energy density is $u$. In that respect, such conversion process appears more efficient the more the ratio $\left(t_{P} / \Delta t\right)$ increases.

\section{Discussion and Conclusions}

In this paper we have considered the process of particle creation from the vacuum state of a massless scalar field enclosed in a small cavity which undergoes a rapid transition from being at rest in a static gravitational field to a state of free fall. The number density $\varrho$ and the energy density $u$ of the created quanta in the reference frame of a comoving observer have been computed by means of the $\beta$-Bogolubov coefficients following a perturbative approach.

The results show that the creation effect involves the gravitational acceleration $\gamma_{0}$. This was basically expected as-in some respect—-the physical scenario recalls the Hawking and Unruh effects, both controlled by an acceleration parameter. However, in the present case, the comoving observer is not eternally accelerated. Instead, the observer's noninertial state suddenly changes into an inertial one, during the subsequent free-fall stage.

Due to such a transition, the particle creation effect depends also on a further parameter $\alpha$, describing the time duration of the transition from the rest to the free-fall state. Actually, as we can see looking at (50), both the above-cited parameters, $\gamma_{0}$ and $\alpha$ enter in defining, e.g., the total energy density $u$ of the created field quanta.

Assuming the specific acceleration profile (6), we obtained an energy spectrum that recalls the one pertaining to a two-dimensional blackbody, whose equivalent temperature is not determined by the acceleration parameter $\gamma_{0}$ (as in the Hawking-Unruh temperature) but by the $\alpha$ parameter ( $c \alpha$, in SI units), representing the so-called jerk (i.e., the second time derivative of the velocity). Although the shape of the energy spectrum is expected to depend on the details of the function $\gamma(t)$ (see (6)), describing the way the cavity is dropped, it is likely that the spectrum becomes more and more independent of the details of $\gamma(t)$, as the transition time $\Delta t \rightarrow 0$, thus yielding $\gamma(t) \rightarrow \gamma_{0} \Theta(-t)$. This suggests that, in that limit, the obtained results hold true quite generally.

Last, but not least, a bit of manipulation of (50) allowed us to express the energy density of the created quanta in terms of the energy density $w_{g} \equiv t_{t t}$ of the background gravitational field. Although such a result has been derived in the weak field limit, hence 
making use of the approximated form of the gravitational energy pseudo-tensor $t_{\mu v}$, it is suggestive that the conversion of gravitational energy into the energy of created field quanta is controlled by the ratio $t_{p} / \Delta t$, according to (51).

An interesting issue concerns the Lorentz Invariance. In Minkowski spacetime, the Lorentz symmetry plays a fundamental role in the existence of the Unruh effect, as shown, e.g., in [29]. This is basically due to the necessity to boost the Unruh detector continuously (at a constant acceleration) in a Minkowski background.

In the present case, involving a curved background, Lorentz Invariance becomes a piece of the Einstein Equivalence Principle, yet restated in its local form. Namely, Local Lorentz Invariance (LLI) states that the outcome of any local nongravitational experiment is independent of the velocity of the freely-falling reference frame in which it is performed.

The analysis we carried on concerning the dynamical evolution of a quantum field confined to a cavity which is suddenly dropped in free fall, represents a gravitational experiment, at least until the cavity has reached its final stage of free fall. Indeed, during the lapse of time required to take the cavity from rest in the static gravitational field to the final stage of free fall, one is at liberty to choose quite arbitrarily the function $\gamma(t)$, describing the way the cavity is dropped, hence obtaining different spectra of created field quanta. In that respect, different observers, each comoving with a cavity (obviously, we assume identical cavities) whose transition to free fall is described by a different $\gamma(t)$, will experience different numbers of created particles. This is not too surprising, since the particle concept is indeed observer-dependent (see, e.g., [15]).

As regards the LLI violation, there is however an interesting point involving Equation (51), in which the Planck time appears. That latter quantity is not a Lorentz invariant, being related to the Planck length which is not an invariant quantity (LorentzFitzGerald contraction), and could be a possible cause of LLI violation at some level in quantum gravity (see, e.g., Will [30]). In that respect, the present analysis could representat least on theoretical grounds-a possible test of LLI violation at the Planck scale. Notice, however, that in the limit of $\Delta t \rightarrow 0$, the number density becomes independent of the details of the cavity transition from rest to free fall (hence restoring LLI), although such a case requires a regularizing cutoff to deal with the divergence in the energy density of the created particles. We expect that in such a limit, the LLI would be recovered.

While, in commonly encountered physical conditions, the amazing smallness of the ratio $\left(t_{P} / \Delta t\right)$ in (51) unavoidably precludes any chance of experimental detection, the obtained results suggest that particle creation in confined quantum systems could become relevant and of some interest in suitable astrophysical and cosmological scenarios, involving rapid changes in the background geometry, as perceived by a confined quantum field. This could be, e.g., the case of an inflationary phase in the early universe.

Finally, we have to bear in mind that we worked out a simplified model, discarding, e.g., possible edge and finite-size effects. Furthermore, the present analysis has been rooted on a perturbative approach. In that latter respect, a deeper investigation involving extreme physical conditions, in which back-reaction effects could play a relevant role, will presumably require nonperturbative techniques. This, we hope, will be the goal of a future investigation.

Funding: This research received no external funding.

Institutional Review Board Statement: Not applicable.

Informed Consent Statement: Not applicable.

Data Availability Statement: Not applicable.

Acknowledgments: I would like to thank the Referees for their useful comments and suggestions, which helped me to improve the manuscript.

Conflicts of Interest: The author declares no conflict of interest. 


\section{References}

1. Parker, L. Particle Creation in Expanding Universes. Phys. Rev. Lett. 1968, 21, 562-564. [CrossRef]

2. Hawking, S.H. Particle creation by black holes. Comm. Math. Phys. 1975, 43, 199-220. [CrossRef]

3. Unruh, W.G. Notes on black hole evaporation. Phys. Rev. D 1976, 14, 870-892. [CrossRef]

4. Akhmedov, E.T.; Singleton, D. On the physical meaning of the Unruh effect. Pis'ma v Zhurnal Èksperimental'noi i Teoreticheskoi Fiziki 2007, 86, 702-706. [CrossRef]

5. Cozzella, T.; Landulfo, A.G.S.; Matsas, G.E.A.; Vanzella, D.A.T. Proposal for observing the Unruh effect with classical electrodynamics. Phys. Rev. Lett. 2017, 118, 161102. [CrossRef]

6. Moore, G.T. Quantum theory of the electromagnetic field in a variable-length one-dimensional cavity. J. Math. Phys. 1970, 11, 2679-2691. [CrossRef]

7. Dodonov, V.V.; Klimov, A.B.; Nikonov, D.E. Quantum phenomena in nonstationary media. Phys. Rev. A 1993, 47, 4422-4429. [CrossRef]

8. Dodonov, V.V. Current status of the dynamical Casimir effect. Phys. Scr. 2010, 82, 036105. [CrossRef]

9. Dodonov, V.V. Fifty Years of the Dynamical Casimir Effect. Phys. Rep. 2020, 2, 67-104. [CrossRef]

10. DeWitt, B.S. Quantum field theory in curved spacetime. Phys. Rep. 1975, 19, 295-357. [CrossRef]

11. Davies, P.C.W.; Fulling, S.A. Radiation from a Moving Mirror in Two Dimensional Space-Time: Conformal Anomaly. Proc. R. Soc. Lond. A. 1976, 348, 393-414.

12. Davies, P.C.W.; Fulling, S.A. Radiation from moving mirrors and from black holes. Proc. R. Soc. Lond. A. 1977, 356, $237-257$.

13. Good, M.R.R.; Anderson, P.R.; Evans, C.R. Time Dependence of Particle Creation from Accelerating Mirrors. Phys. Rev. D 2013, 88, 025023. [CrossRef]

14. Lock, P.E.; Fuentes, I. Dynamical Casimir effect in curved spacetime. New J. Phys. 2017, 19, 073005. [CrossRef]

15. Birrell, N.D.; Davies, P.C.W. Quantum Fields in Curved Space; Cambridge Monographs on Mathematical Physics; Cambridge University Press: Cambridge, UK, 1984.

16. Sorge, F.; Wilson, J.H. Casimir effect in free fall towards a Schwarzschild black hole. Phys. Rev. D 2019, 100, 105007. [CrossRef]

17. Wilson, J.H.; Sorge, F.; Fulling, S.A. Tidal and nonequilibrium Casimir effects in free fall. Phys. Rev. D 2020, 101, 065007. [CrossRef]

18. Manasse, F.K.; Misner, C.W.T. Fermi Normal Coordinates and Some Basic Concepts in Differential Geometry. J. Math. Phys. 1963, 4, 735-745. [CrossRef]

19. Marzlin, K.-P. Fermi coordinates for weak gravitational fields. Phys. Rev. D 1994, 50, 888-891. [CrossRef]

20. De Felice, F.; Bini, D. Classical Measurements in Curved Space-Times; Cambridge Monographs on Mathematical Physics; Cambridge University Press: Cambridge, UK, 2010; pp. 80-82.

21. Buchholz, D.; Verch, R. Macroscopic aspects of the Unruh effect. Class. Quantum Gravity 2015, 32, 245004. [CrossRef]

22. Born, M. Die Theorie des starren Elektrons in der Kinematik des Relativitätsprinzips. Ann. Phys. 1909, 335, 1-56. [CrossRef]

23. Born, M. Über die Dynamik des Elektrons in der Kinematik des Relativitätsprinzips. Phys. Z. 1909, 10, 814-817.

24. Sorge, F. Casimir effect in a weak gravitational field. Class. Quantum Gravity 2005, 22, 5109-5119. [CrossRef]

25. Cardoso, T.R.; de Castro, A.S. The Blackbody Radiation in D-Dimensional Universes. Revista Brasileira de Ensino de Física 2005, 27, 559-563. [CrossRef]

26. Davies, P.C.W. Scalar production in Schwarzschild and Rindler metrics. J. Phys. A Math. Gen. 1975, 8, 609-616. [CrossRef]

27. Gradshteyn, I.S.; Ryzhik, I.M. Table of Integrals, Series and Products; Elsevier Academic Press: Burlington, VT, USA, 2007.

28. Landau, L.D.; Lifshitz, E.M. The Classical Theory of Fields; Pergamon Press: Oxford, UK, 1975.

29. Campo, D.; Obadia, N. Why does the Unruh effect rely on Lorentz invariance, while Hawking radiation does not? arXiv 2010, arXiv:1003.0112v1.

30. Will, C.M. The Confrontation between General Relativity and Experiment. Living Rev. Relat. 2014, 17, 4. [CrossRef] 